\title{
Development of Self Speaking Body Weight Scale for Visually Impaired People in Tanzania
}

\author{
Gloriose Nzasangamariya ${ }^{1}$, Ramadhani Sinde ${ }^{2}$, Shubi Felix Kaijage ${ }^{3}$ \\ Research Scholar ${ }^{1}$, Lecturer ${ }^{2}$, Senior Lecturer ${ }^{3}$ \\ Embedded and Mobile Systems
}

Nelson Mandela African Institution of science and technology

Arusha, Tanzania

\begin{abstract}
Speaking weight scale is an important low vision health aid that measures and announces the measured weight. It is valuable in numerous applications such as Bathroom scale, Kitchen scale, and more. Different talking scales have been developed for the blind community. Many talking scales have language options for English, German, French, or Spanish. However, only limited work exists for Self speaking visually impaired community in EAC given the fact that no talking scale can announce weight in Self which is the common language in EAC. Therefore, this project aims to develop a Self-speaking weighing machine to assist visually impaired people in Tanzania. The developed device is divided into two major parts. On the front end of the design, sensors are used to capture weight parameters. The captured values are mapped onto a sequence of voice patterns. The back-end consists of transferring a sequence of voice patterns to a loudspeaker whereby the voice patterns are stored on an SD card. Finally, the developed device has been evaluated on several objects with known weights. The results show that the developed device accurately measures weight, displays weight, and announces it in the Self language. However, blind people still need assistance from sighted persons to be directed to the scale's platform. The developed device has great potential as a low vision health aid for Self speakers. Moreover, the features of this device can be further improved by integrating iBeacon technology to increase the autonomy of blind people to use the scale and navigate to the device's location safely.
\end{abstract}

Key words: Self speaking, Speaking weight scale, Visually impaired people.

\section{INTRODUCTION}

Statistics has estimated that "eighty to eighty-five percent of our perception, learning, cognition and activities are mediated through vision". The millions of blind people worldwide are at a major disadvantage compared to sighted individuals. For example, using bathroom scale to weigh themselves, using kitchen scale to weigh ingredients when cooking is a simple set of tasks for a sighted person. However, for a blind person these tasks present a series of challenges. Controlling body weight, even a civilized one, is almost impossible for an individual with a severe visual impairment without some kind of assistive tool.

Assistive device like existing talking scales do not provide any assistance to Self speaking visually impaired community. Numerous speaking scales available on the market are mostly useful tool for people who understand western languages such as English, Spanish, Greek, German. There is always a question mark for Self speaking visually impaired people and Self speaking community at large to interpret results of existing talking scales. The same limitation applies to mechanical and traditional weighing scales used in marginalized areas in Tanzania due to the fact that they cannot be used by a person with visual impairment. Tradition scales consist of two plates placed at equal distances from a support. One plate curries the object to be measured while other plate curries the predetermined weight. At the end the weight on both plates should be equal [1]. 
The purpose of this paper is to develop a speaking body weight scale which measures and announces weight in Kilograms in Self language and provides digital output on the Liquid Crystal Display (LCD) screen which provide efficient and seamless means of which to control weight for their healthy life for Self speaking people living with low vision of all levels as it is recommended particularly in health sector to do regular self-weighing for all people to live healthy life [2]. This scale sends its measurements to the smartphone within vicinity to accommodate people with mobility limitations which prevent them from viewing the scale readout in traditional manner, the scale measurements can then be accessed through the smartphone within the vicinity.

This paper explains the design and implementation of Self Speaking Body Weight Scale gadget by using speech technology and strain gauge load cell technology. The weighing system is incorporated within a mobile application and smartphones within vicinity receive information from a weighing scale to speak out the scale readout. The implementation of this Self speaking weighing machine is carried out at Nelson Mandela African Institution of Science and Technology, which is a research institution for postgraduate and post-docs studies and research in Science, Engineering, Technology and Innovation (SETI) in Arusha- Tanzania.

\section{LITERATURE SURVEY}

Speech technology is increasingly being used in electronic devices to make them accessible to blind or low vision persons [3]. Talking scale is one of emerging speech enabled devices needed by visually impaired people particularly in health sector where regular selfweighing is recommended for all people to improve adaptation of weight control to stick to healthy habits in long run [4].

Talking scale has numerous applications such as Bathroom scale for measuring body weight, Kitchen scale for measuring ingredients when cooking. It can also be used in grocery stores and more. For many of these applications, most of the basic operations remain the same.

\subsection{Existing talking scales}

Talking weighing scale is a low vision health aid which measure and announces weight of something being measured. Talking scale speaks out the measurements loud to people in a clear voice whereby people do not need to bend themselves to check the scale' $\mathrm{s}$ measurements on the display. Many talking scales have a language option for English, German, French or Spanish.

In the design of Talking scale, the main hardware used in the processes includes load cells sometimes called weight sensors. These are transducers which convert mechanical energy into electrical energy whereby the produced electrical signal is directly proportional to the weights of objects being measured (Load Cell Central, 2020) [5]. There is also a microcontroller which is a processing unit. It gathers data from weight sensors, processes them, displays digital output on Liquid LCD screen and then convert displayed weight into audio using text to speech technology [6].

\subsection{Proposed work}

Self Speaking Body Weight, it is just like other talking scale however it is based on Self language and sends measured weight to a mobile application to be accessed through smartphones. Typically, Self Speaking Body Weight Scale uses stored audio files on SD card. SPI interface offers a communication between SD card and microcontroller. The integrated circuit within the microcontroller offers the necessary intelligence circuitry for computation purposes, for instance, the circuitry computes the data from weight sensors (load cells). Based on the output produced, it then fetches corresponding audio file from SD card. The fetched audio is forwarded to the audio amplifier then to the loudspeaker. 


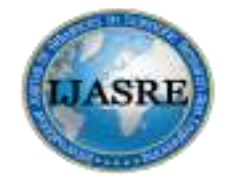

\section{International Journal of \\ Advances in Scientific Research and Engineering (ijasre)}

DOI: 10.31695/IJASRE.2021.34044
E-ISSN : 2454-8006

Volume 7, Issue 7

July - 2021

\subsection{Purpose and motivation}

The main purpose of this device is to offer Self speaking visually impaired people an efficient and seamless means of which to control their weight for their healthy life, increase awareness of people to measure their weights and at the same time embrace the development of technology in Self language. This weighing gadget also aims to provide convenience to people from marginalized area in Tanzania while solving the drawbacks of existing weighing machines which are being used in rural areas. Beside this a great percentage of the population in urban areas are individuals with a lot of responsibilities and do not need to be present to view the scale readout from the standing angle. There is also a good percentage of individuals who have mobility limitations and depend on others to tell them the scale measurements, something of which is not always very reliable. And this is intended to be solved by the integration of a talking weighing system with a mobile application to speak out the scale measurements on a smartphone. The usefulness of this gadget is further justified by its applicability in other fields like Kitchen scale for measuring ingredients when cooking, in grocery stores and many more.

\section{METHODOLOGY}

This project is divided into two major parts. On the front-end of the design, sensors are used to capture physical parameters such as weight and the captured values will be mapped onto a sequence of voice patterns. The back-end of the design consists of transferring a sequence of voice patterns to a loudspeaker, where the voice patterns are stored on an SD card as .WAV files. voice patterns are then transferred to the amplifier and forwarded to the loudspeaker.

Processes of implementation

Figure 1 shows physical connections among different main hardware devices used in the design.

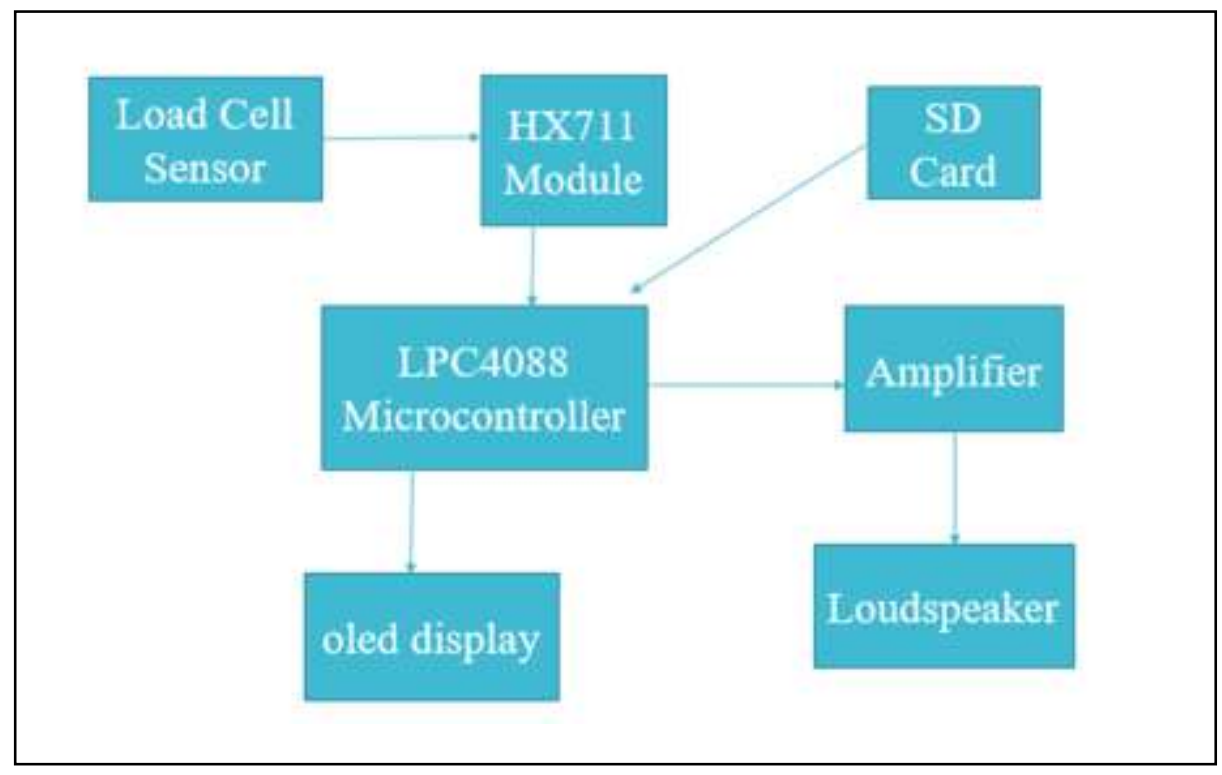

Figure 1: Hardware Block Diagram of the design

\subsection{Interfacing Load cell with HX711 module}

Load cell: This is a weight sensor which uses strain gauge technology. It works by changing force/weight of objects being measured into electrical resistance whereby the produced electrical resistance is proportional to the stress/strain of an object being measured making it easy to calibrate into an accurate measurement [7].Using strain gauge load cell was best suited for it is specialized for weighing devices, given that it has better accuracy and precision. 
HX711: This module serves the purpose of amplifying the low output produced by weight sensors at the same time servers as Digital to Analog Converter (ADC) and sends sensor's data to the microcontroller [8]. HX711 was best suited for it is specialized for weight scales.

For a single scale, 4 load cells were used as weight sensors. Each load cell has 3 wires which are: White wire, black wire and red wire. Each load cell measures up to $50 \mathrm{Kgs}$ maximum. All the 4 load cells are connected in a loop by connecting together white to white cables and black to black cables the neighbouring load cells. One diagonal red wires are used as power wires. They are connected to $\mathrm{E}+$ and E- output pins of HX711 amplifier module. E is the excitation voltage, E+ is the excitation positive and E- is the excitation ground. HX711 module is supplied by the microcontroller itself. Hence E+ and E- are supplied through the circuit internally. The other diagonal red wires are used to take measurements from sensors (load cells). They are connected to A+ and A- input pins of HX711 amplifier module. A+ and A- are sensor values. Since all 4 Load cells are connected in a loop then A+ and A- takes the measurements from all the 4 sensors and send to HX711 module' s inputs.

Figure 2 shows the connection between Load cells and HX711 module.

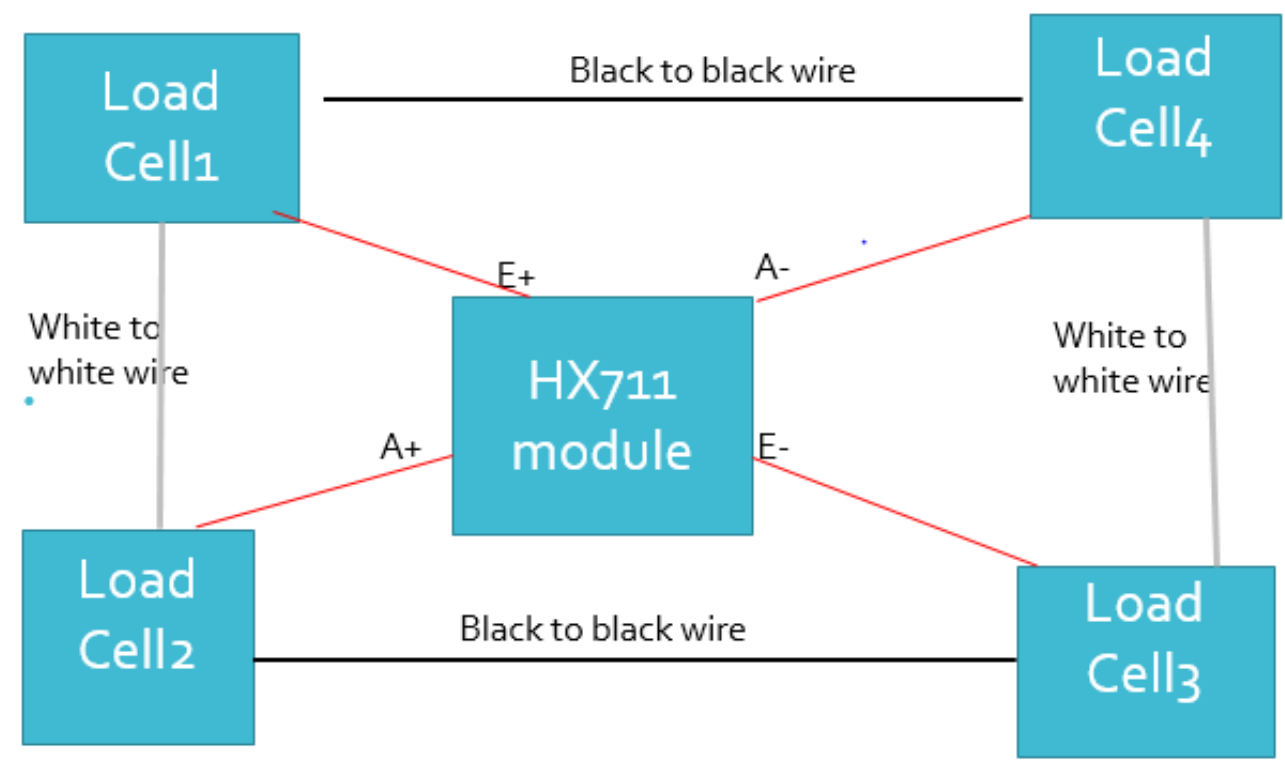

Figure 2: Fig. 2. Interfacing load cell with HX711 module

After connecting all the 4 load cells to the HX711 module, HX711 module was then interfaced with Esp8266 NodeMCU microcontroller over serial interface. Esp8266 NodeMCU was used as a processing unit. It receives data from HX711 module, computes them, displays digital output on the screen and call WAVE files from Secure Digital (SD) card which correspond to the produced out and finally forwards digital audio signal to the audio amplifier.

Esp8266 NodeMCU was then interfaced with SD card over Serial Peripheral Interface (SPI) interface. Secure Digital (SD) Card is a low cost non-volatile memory card used in mobile devices to store data and to enable the transfer of data between devices. $32 \mathrm{~GB}$ micro SDHC card was used to store WAVE files and was formatted as FAT32. Finally, Esp8266 NodeMCU was interfaced with audio signal with Max98357A Inter-Integrated Circuit Sound (I2S) audio amplifier over I2S interface.

Audio data of weight voices in Self is stored on SD card as .WAV file and sent out over the I2S port of Esp8266 NodeMCU to Max98357A I2S audio amplifier and forwarded to the loudspeaker.

I2S (Inter-Integrated Circuit Sound) interface is the standard serial interface protocol for transferring audio data between devices.

Audio data is stored on SD card as .WAV file and sent out over the I2S port of Esp8266 NodeMCU to Max98357A I2S audio amplifier and forwarded to the loudspeaker. Here Esp8266 NodeMCU is acting as a master since it is generating bit clock, word-select signal and data.

After having all the hardware connected together, the second step was to prepare audio data. Audio data was sampled using python language and stored on an SD card with .WAV extension. Each weight number was stored separately as an individual wave file. For instance, .WAV file of kilo moja, .WAV file of Kilo mbili, .WAV file of Kilo tatu and so on.

After having audio data in place, the system which measures weight and displays scale readout on LCD screen was developed using 


\section{International Journal of Advances in Scientific Research and Engineering (ijasre), Vol 7 (7), July -2021}

Arduino Integrated Development Environment (IDE) and HX711 libraries.

EEPROM library was used in Arduino codes to save calibration parameters or calibration index of the scale. Oled: This library was used for display and uses Inter-Integrated Circuit (I2C) protocol to interface ESP8260 microcontroller with Oled display.

Finally, the mobile application was developed using android. ESP8260 Wi-Fi library was used to enable ESP8260 nodemcu in wireless communication. WIFI User Datagram Protocol (UDP) library was used to allow ESP8260 nodemcu to use UDP functions when it is sending data to the smartphone. For a smartphone within vicinity to communicate with weight scale, user has to setup Wi-Fi hotspot whose credentials are the same as the ones configured with the weight scale in Arduino codes. The scale uses UDP socket to broadcast data to the authorized smartphone within vicinity. Figure 3 shows an overview of the weight announcement process.

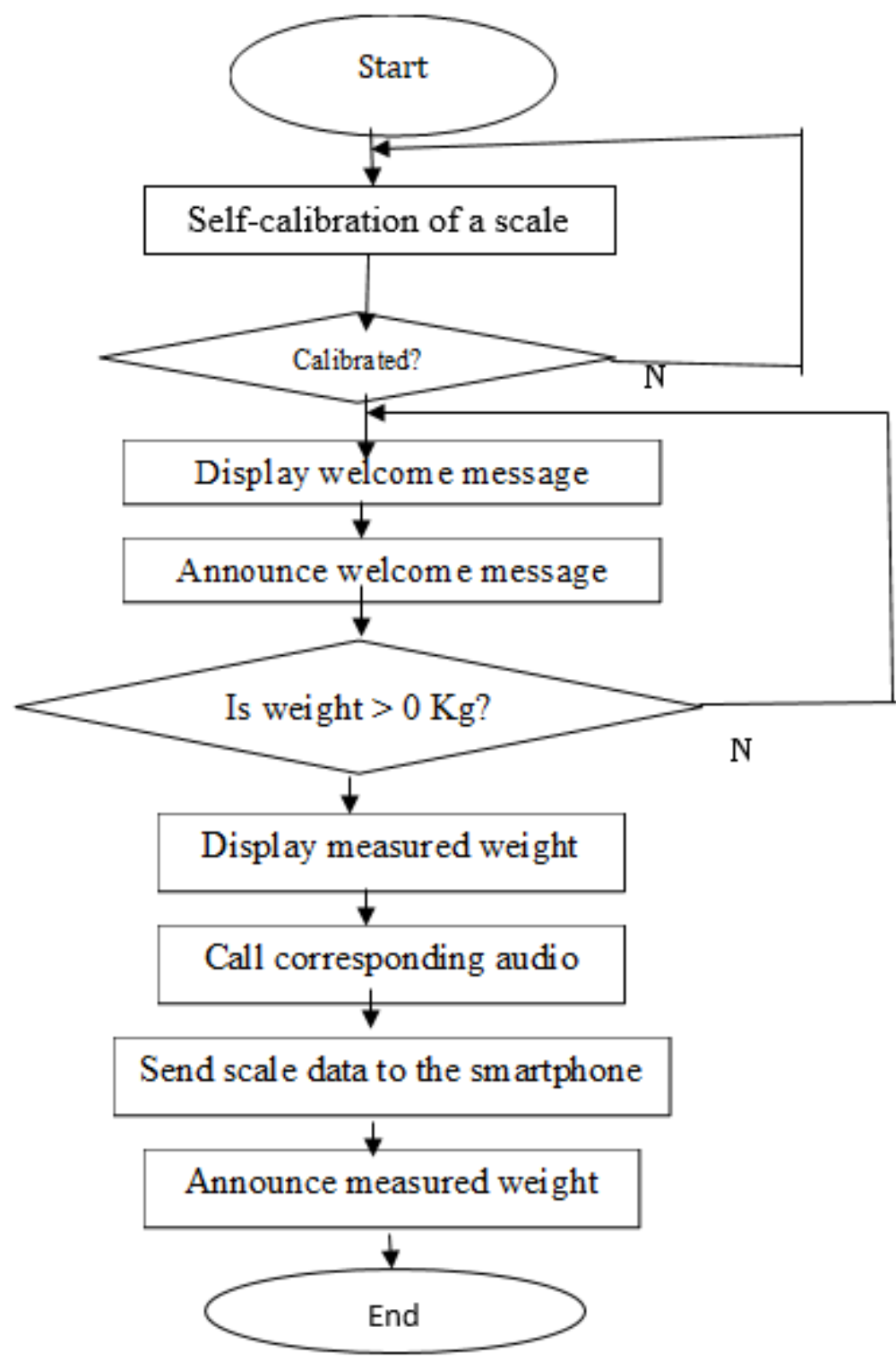

Figure 3: Flowchart of weight announcement 


\section{RESULTS AND DISCUSSION}

A prototype for Self speaking Body Weight Scale was physical designed and all hardware worked as intended. The main hardware components used are 4 pieces of 50kg Load cell, HX711 amplifier module, Esp8266 NodeMCU, oled1.3-inch display, Max98357A I2S audio amplifier and 32GB micro SDHC card. Esp8266 NodeMCU was used with a voltage regulator to convert $5 \mathrm{~V}$ to $3.3 \mathrm{~V}$ as power supply provides $5 \mathrm{~V}$ and Node mcu (esp8266) uses 3.3V.

The proposed speaking scale has been evaluated on several objects with known weights. The results show that the developed device successfully performs key functionalities like to accurately measure weight, display weight and announce it verbally in Self language. Despite the fact that the device performs the key functionalities, blind people still need assistance from sighted person to be directed to the scale's platform safely. Therefore, some improvements need to be made.

Fig. 4 shows the constructed working prototype.

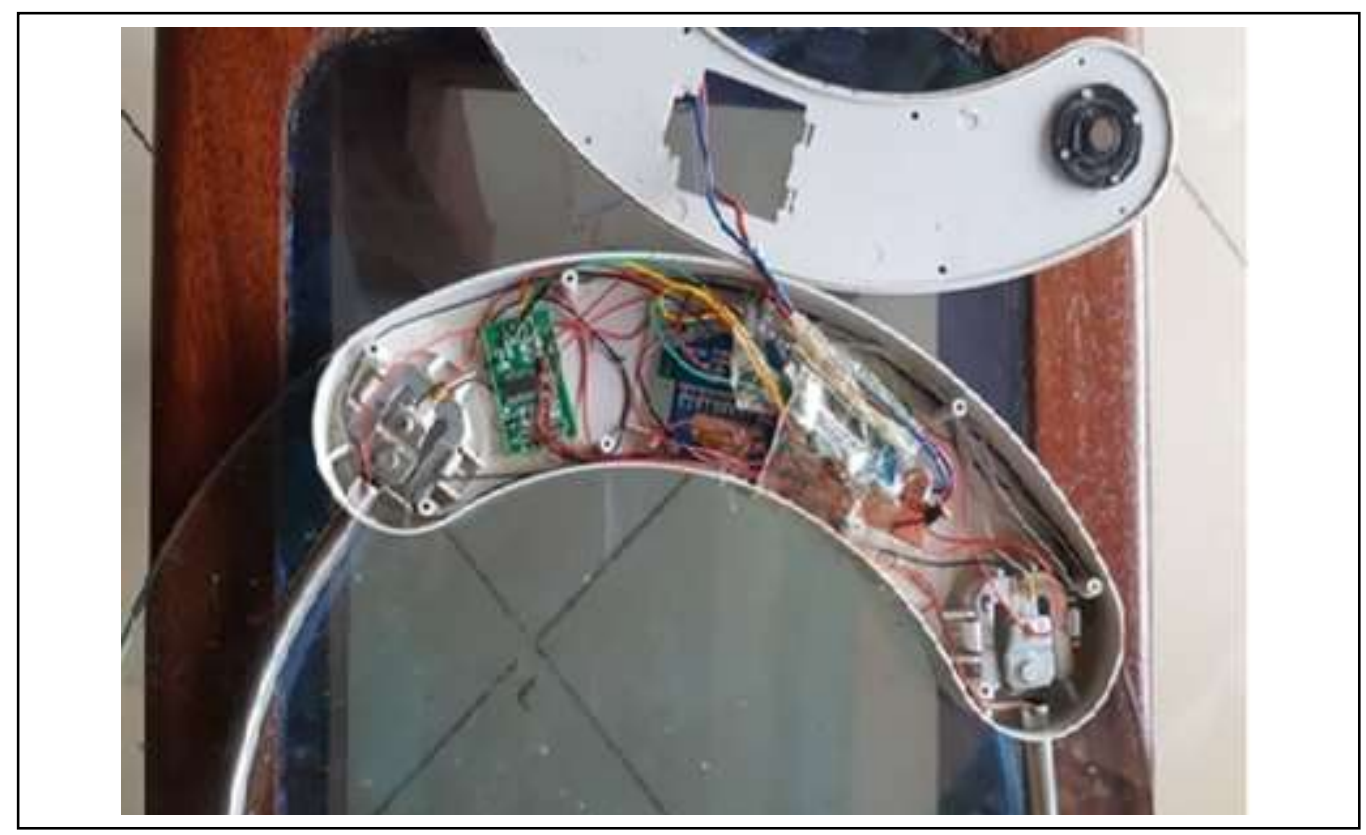

Figure 4: Prototype of the design

Figure 5, 6 and 7 below show the discussed results

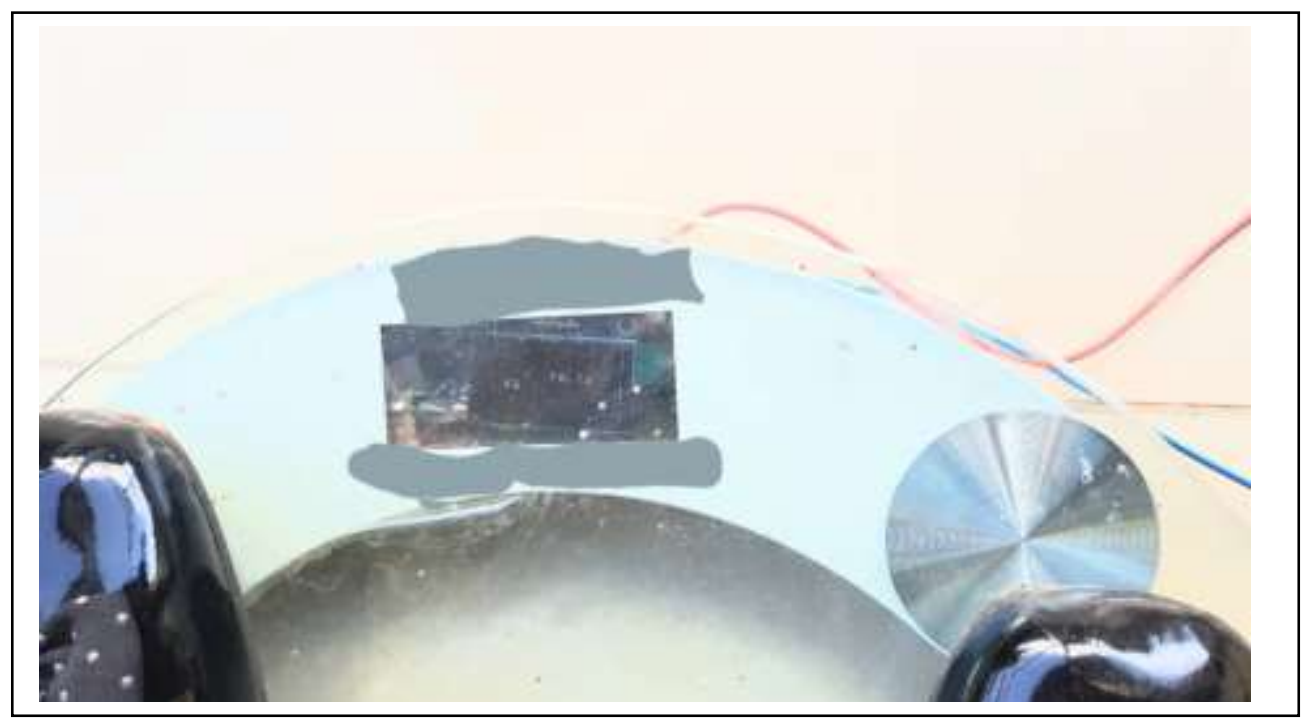

Figure 5: Scale readout on the display 
International Journal of Advances in Scientific Research and Engineering (ijasre), Vol 7 (7), July -2021

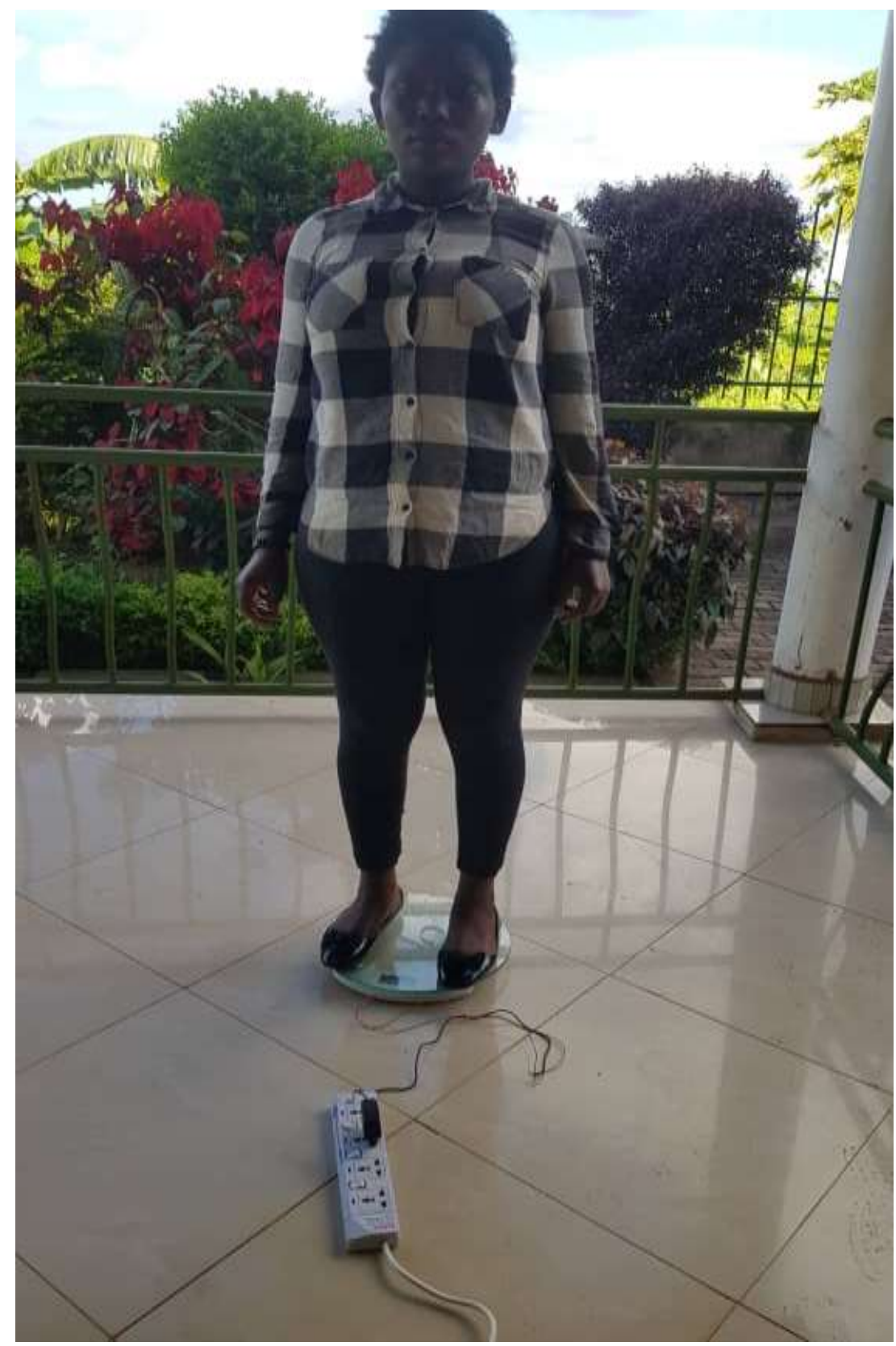

Figure 6: Picture showing a visually impaired person using the scale 


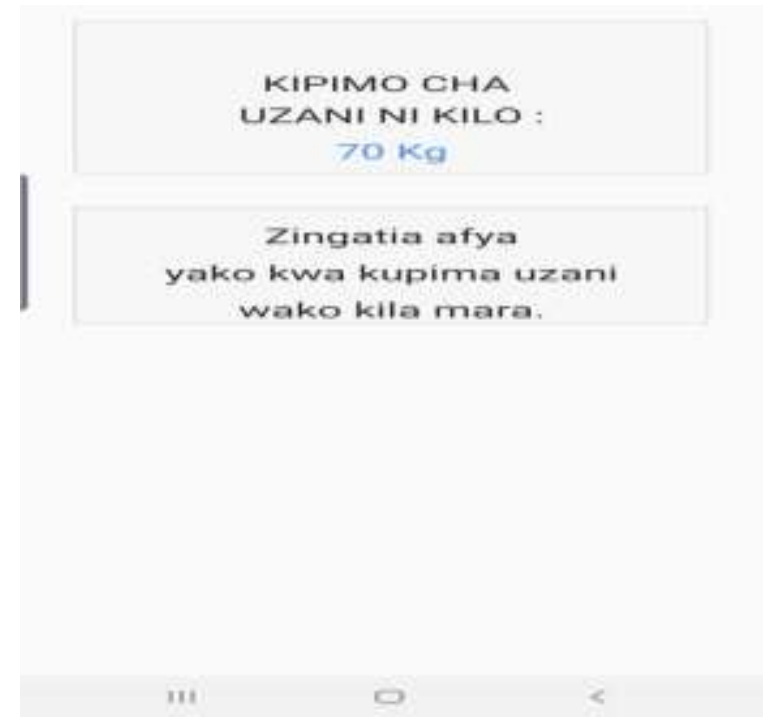

Figure 7: screenshot of scale' s data sent to the smartphone

\section{CONCLUSION}

This project aimed to raise people awareness of controlling their weight and hence live healthy life as well as to encourage the adaptation of development of technology in Self language by developing a Self speaking body weight machine. The project focused on Self speaking instrument due to its significance in providing access of measuring devices to Self speaking visually impaired people and due to the fact that visually impaired people receive little consideration especially in third world countries when new technology is introduced as many advanced technologies are limited to developed countries for instance no talking scale can announce weight in Self language.

This study evaluated the current body talking weight machines and found that one of the research gaps is ignorance in weight control for people living with visual impairment in Tanzania. After collecting and analysing needs, perceptions and recommendations of visually impaired people on having Self speaking weight machine to offer Self speaking visually impaired people an efficient and seamless means of which to control their weight for their healthy life as regular self-weighing is recommended to all people.

Despite the fact that the developed device has a great potential as a low vision health aid for people living with vision impairments for health and weight track, more research is open to further improve features of this device by integrating iBeacons technology to allow blind people to navigate to the device's location safely [9]. In addition to this a device can be improved to send data to the cloud whereby all concerned personnel can access the scale measurements remotely.

\section{REFERENCES}

[1] D. Morse and D. M. Baer, "Laboratory Balances: How They Work , Checking Their Accuracy," doi: 10.1309/QYR5UV73FRY2YBMJ.

[2] J. J. VanWormer, S. A. French, M. A. Pereira, and E. M. Welsh, "International Journal of Behavioral Nutrition and Physical Activity The Impact of Regular Self-weighing on Weight Management: A Systematic Literature Review," Int. J. Behav. Nutr. Phys. Act., vol. 5, no. 5, 2008.

[3] P. Blenkhorn, "Designing products that speak: Lessons from talking systems for blind people," Comput. Control Eng. J., vol. 5, no. 4, 1994, doi: 10.1049/cce:19940402.

[4] R. Bivins and H. Marland, "Weighting for health: Management, measurement and self-surveillance in the modern household," Social History of Medicine, vol. 29, no. 4. 2016, doi: 10.1093/shm/hkw015.

[5] P. Hambir, N. Joshi, P. Karande3, and A. Kolhe, “Automatic Weighing and Packaging Machine,” Int. Res. J. Eng. Technol., vol. 06, no. 05, 2019. 
[6] A. Rajbongshi, M. I. Islam, M. M. Rahman, A. Majumder, M. E. Islam, and A. A. Biswas, "Bangla optical character recognition and text-to-speech conversion using raspberry Pi," Int. J. Adv. Comput. Sci. Appl., vol. 11, no. 6, 2020, doi: 10.14569/IJACSA.2020.0110636.

[7] P. K. H. Thakkar, "Performance Evaluation of Strain Gauge Based Load Cell to Improve Weighing Accuracy," Int. J. Latest Trends Eng. Technol. Perform., vol. 2, no. 1, 2013.

[8] E. A. Akindele, O. V. Matthews, and K. O. Idowu, "Development of an Electronic Weighing Indicator for Digital Measurement," Int. Res. J. Eng. Technol., vol. 5, no. 9, 2018.

[9] B. Li et al., "Vision-Based Mobile Indoor Assistive Navigation Aid for Blind People," IEEE Trans. Mob. Comput., vol. 18, no. 3, 2019, doi: 10.1109/TMC.2018.2842751.

\section{Gloriose Nzasangamariya ${ }^{1}$}

Email: nzasangamariyag@nm-aist.ac.tz 